\title{
Continue with ICU care - she is a spiritual being
}

\author{
NICO NORTJÉ, KAREN N TERRELL
}

\begin{abstract}
This case study discusses a dispute between the healthcare team and the patient's surrogate decision maker at a cancer centre. While the healthcare team deemed further care to be futile, the patient's husband argued that they should continue to try to reverse his wife's acute decline. This case study illustrates the inertia and moral distress that can result when there are differences between patients/surrogates and the healthcare team in their goals for intensive care. The issues of moral distress and an inability to make decisions were addressed by involving an ethics consultant, and by creating institutional mechanisms to address end-of-life issues at an earlier stage.
\end{abstract}

Keywords: futile care, surrogate decision-making, advance care planning, end of life care, moral distress

\section{Case study}

Mrs BM was a 59-year-old female suffering a relapsed blood cancer. Treatment for this condition usually includes a combination of chemotherapy and stem-cell transplantation. Patients are sometimes entered into clinical trials for investigational treatments. Mrs BM received various lines of therapy but still had persistent disease. While her oncology team was looking into investigational chemotherapy options, she developed pain and swelling in her upper left arm. Mr BM brought his wife to the emergency centre, where it was found that she had a fever, sepsis, acute kidney injury, a high level of potassium in the blood, and severe metabolic acidosis (an imbalance of the electrolytes in the body). As the emergency centre physician was busy with her work-up, Mrs BM's heart stopped beating. She was resuscitated and transferred to the

Authors: Nico Nortjé (corresponding author - nnortje@mdanderson.org), Section of Integrated Ethics, Department of Critical Care, The University of Texas MD Anderson Cancer Center, Houston, TX, USA and Center for Healthcare Ethics, Lakehead University, Thunder Bay, ON, CANADA; Karen N Terrell (kterrell@mdanderson.org), Section of Integrated Ethics, Department of Critical Care, The University of Texas MD Anderson Cancer Center, Houston, TX, USA.

To cite: Nortje N, Terrell KN. Continue with ICU care - she is a spiritual being. Indian J Med Ethics. Published online on August 18, 2021. DOI: 10.20529/IJME.2021.063

Manuscript Editor: Olinda Timms

Peer Reviewers: Raj Kumar Mani and an anonymous reviewer

(c) Indian Journal of Medical Ethics 2021 intensive care unit (ICU), put on a ventilator, and started on four medications to regulate her blood pressure. Unfortunately, she had altered mental status, which was believed to have been caused by a lack of oxygen to the brain, and could not participate in decision-making. Mr BM became his wife's surrogate decision-maker and elected to keep her code status as full code, necessitating the care team to provide resuscitation should Mrs BM's heart stop again. She was also passing large blood clots, probably from bleeding in her gastrointestinal tract. Owing to these complications, it was clear to the ICU care team that recovery to her previous condition was unlikely.

Prior to the onset of Mrs BM's acute events, her healthcare team had initiated advance care planning (ACP) conversations with her and her spouse, as a routine intervention in the care of cancer patients. ACP involves ongoing conversations between healthcare professionals, patients, and the patients' loved ones. The objective of these conversations is to communicate to the healthcare team the patients' wishes, values, beliefs, and goals for long-term, future medical treatment and care in advance of a medical crisis (1). Unfortunately, patients are not always receptive to these conversations and are often more focused on a cure and escalation of treatment options. Healthcare professionals are encouraged to initiate ACP conversations with patients early in the course of treatment in order to maintain the patient's autonomy, improve their quality of life, and improve patient and family satisfaction (2). However, in this case, Mrs BM did not have these conversations with the care team and at some point clearly communicated her wishes only to Mr BM. The healthcare team was left facing challenges when trying to align care goals with standard practices and Mrs BM's (and her husband's) wishes.

The sudden onset of Mrs BM's decline was distressing to $\mathrm{Mr}$ $\mathrm{BM}$, who had been preparing to bring his wife in for another round of therapy, not for life-sustaining measures. Mr BM was a highly educated and well-read person. His relationship with his wife was more than just physical; they were spiritual partners as well. To this effect, he shared his wife's wishes with the care team saying, "It's fine if her body doesn't function. As long as her mental acuity is still present, it will be quality of life for her." These sentiments are common among family members of ICU patients; especially when the onset of the unexpected event is rapid, families often prefer to opt for 
aggressive treatments and to "give it a shot." If a surrogate decision maker or family member "gives up too early," it can be seen as not trying or not advocating for the patient. Studies indicate that the emotional distress experienced by family members of ICU patients when they need to make difficult decisions often results in severe post-traumatic stress syndrome, also referred to in the literature as family intensive care unit syndrome (3).

Healthcare professionals are encouraged to have a structured approach to facilitating ACP conversations, thereby alleviating barriers to having such conversations (1). However, even after creating a structured approach, most physicians feel that leading these conversations could lead to loss of hope, emotional distress, and impaired professional relationships between the patient, the family unit, and the healthcare team.

The care team consulted with the hospital's ethicists about 10 days after Mrs BM's admission, as there was a misalignment between what medicine can do and what the patient's (her surrogate's) wishes were for treatment. The role of the ethics consultant is to get a better understanding of the patient's (and surrogate's/family's) context. These include their value systems, what they see as important in life, their spiritual or religious outlook, their support systems, and any other crises they are experiencing (which often translate into existential anxiety - the inability to make choices freely). ACP conversations focus on future changes that may potentially occur and what a patient's current views are of that future event. The aim of an ethics consult however is when two parties (in this case the $\mathrm{Mr} \mathrm{BM}$, and the care team) have opposing views of what is the right thing to do, an ethicist will engage with all the relevant parties and discern whether there is common ground to facilitate shared decisionmaking. In the initial interaction with $\mathrm{Mr}$ BM, the ethicist learned that neither he nor his wife adhered to any specific religion; rather, they saw themselves as free-spirited beings, close to nature. These value statements were relevant to the ethics consultant, as it helped him to "build a bridge" between the patient and her husband and the healthcare team. Through facilitated conversation (4), the ethicist was able to get all the members of the care team onto a Zoom call where he explained that Mr BM was scared and not ready to let go of his wife. It became evident during the discussion that Mr BM had transference of grief (where his feelings of loss were expressed in inertia or an inability to make decisions), which resulted in a more complicated state of anticipated grief (which oftentimes exacerbates feelings of loss of control and anger).

Unfortunately, the care team viewed further treatment of Mrs BM as futile or nonbeneficial; she was nonresponsive, exhibiting clear signs of a poor prognosis and general decline. Mr BM's difficulty in dealing with his grief prolonged the situation, causing more stress for everyone involved. Consequently, there was a disconnect between what the care team and what Mr BM viewed as appropriate, with Mr BM arguing for cancer directed treatment in addition to the lifesustaining treatment instituted at the time of admission. It was also becoming evident that the life-sustaining treatment was no longer appropriate. The teams continued to give Mrs BM the care necessary to try and get over acute events, even though taking care of her created moral distress for many members of the nursing team who interacted with her on a regular basis and witnessed her decline, even the breakdown of her skin. In such cases, the care teams often view the decisions of surrogate decision-makers to continue care as inflicting more harm and suffering on a patient, which from the care team's perspective is immoral. Consequently, members of the care team may feel that their personal value system (ie against inducing suffering) is being attacked and separating it from professional values (ie continuing care respecting the surrogate decision-maker's decision, even if it is inappropriate) becomes challenging.

Moral distress is an emotional state often experienced by critical care nurses (5). It occurs when the nurse or other healthcare provider feels that the ethically correct action to take is compromised by the decisions of the patient or their family/decision-makers. Nurses often describe moral distress as emotionally exhausting, painful, and a sense of suffering; they may experience anger, frustration, and sadness (5). They may feel helpless and experience a sense of loss in regard to the quality of care they can offer their patients. Studies have shown that ICU nurses are at high risk for moral distress due to advances in life-support technology, the intense pace of the work environment, and the constant exposure to death (5). These factors have been shown to increase the number of reported cases of moral distress among ICU nurses and related healthcare professionals (1).

It was clear to the ethics consultant that there had been a breakdown in communication between Mr BM and Mrs BM's care team, and that trust had to be restored. The care team was focused on the medical futility of Mrs BM's treatment, while Mr BM argued that there was still emotional value for him to keep on fighting for his wife's life.

Mrs BM's hospitalisation happened at the start of the Covid19 pandemic. Due to hospital policy, Mr BM had to shelter in place at Mrs BM's bedside and was not allowed to leave the hospital owing to the risk of introducing Covid-19 infection. Consequently, he was not able to have the emotional support of friends and family. This isolation took its toll on him, as he began to fixate on the smallest changes in his wife's daily laboratory values and ventilator settings. Any positive change was seen as an improvement and as a sign that Mrs BM would recover. Sadly, Mr BM was in denial of the overall clinical picture of multiorgan failure and general decline.

All ICU patients at our institution are discussed daily at a utilisation meeting. During these meetings, the healthcare team felt not enough progress had been made and expressed their concern about the trajectory of Mrs BM's care and requested that a Medical Appropriateness Review Committee 
(MARC) meeting be scheduled. The purpose of this institutional committee is to review and discuss the opinions of the doctors treating a patient regarding the medical appropriateness of the recommended treatments. The physicians on the committee review the patient's medical records and consider various medical opinions provided by the treating physicians. MARCs are known to be emotionally taxing for everyone involved and are usually the last resort for conflict resolution.

In this case before the MARC could be held $\mathrm{Mr} B M$ acknowledged his great fear of being without Mrs BM and was able to put his decisions into context. The ethicist, together with a social worker, addressed this anticipated grief, which is often manifested as separation anxiety. Through reflective discussions (focusing on happy events in the past and then reflecting on how to approach these same events without his wife), the ethicist-social worker team was able to help Mr BM to develop coping skills and to transfer his fear of being alone to making sure his wife would not suffer anymore and would have a dignified death. Mr BM went through the usual stages of grief (denial, anger, bargaining, depression, and acceptance). It took him another three days to come to terms with the situation, at which time he asked the ICU team to change his wife's code status to DNR, withdraw lifesustaining care and focus only on comfort care. Mrs BM passed away within 30 minutes of the request being implemented.

\section{Discussion}

Such scenarios play out every day in ICUs around the globe. Several ethical issues can be identified in this case. One is whether it is appropriate to expend a month's worth of futile ICU care on a patient with no sign of recovery during a global pandemic that has been exhausting frontline healthcare workers. Another is why sincere discussions were not held earlier on at the onset of hospitalisation, to avoid one million dollars of healthcare expenditures, moral distress in the healthcare team, and tremendous discomfort to the patient, all to allow the family to come to terms with the patient's prognosis. A third issue is specific to the Covid-19 pandemic: how should the hospital handle conflicts in the ICU caused by the need for surrogates to make decisions while in physical isolation from their support networks?

Healthcare practice has, to a large extent, moved away from a paternalistic approach to place patients' or surrogates' wishes at the center of healthcare decision-making. Choices of treatment options often align with the patient's goals of care and wishes for further treatment. However, when there is an ethical dilemma, as illustrated in this case, it is important for an ethicist to assist with the case and give guidance to the healthcare team. Legislation in different parts of the world gives different levels of decision-making authority to healthcare workers to refuse or limit treatment

The jurisdiction of this case has placed a lot of emphasis on patients' and surrogates' choices. As of April 1, 2018, Texas physicians can no longer make changes to a patient's code status without the patient's consent or that of the holder of medical power of attorney (Senate Bill 11) (6). This legislation has made it difficult for the healthcare team to make the decision to stop futile care - a decision that they feel is necessary for the patient -- without risking criminal prosecution. In this case, the healthcare team had to patiently wait for Mr BM to come to terms with his struggle of letting his wife go. Consequently, it was important for the ethicist to "build a bridge" with Mr BM and to understand his context. As discussed earlier, the ethicist did exactly that and kept checking in daily with Mr BM. The focus of these check-ins was to normalise the process of decision-making and to offer emotional support in these times of isolation.

Another lesson to learn from this case is that the medical team needs to understand that their timeline is not necessarily the timeline of the patient/surrogate decisionmaker. Furthermore, physical isolation causes individuals to hold on to hope, even if, in the bigger picture, that hope is not realistic. As healthcare workers, we have a duty to understand where our patients and their surrogates are in their contextual reality and understanding, and to meet them on their journey. The authors hold that the services of the ethicist should have been requested earlier; this might have helped to alleviate some of the moral distress experienced by the team.

Just prior to this case's end, a Goals of Care Rapid Response Team (GOC RRT) model was developed at the author's institution by a group of stakeholders (including the ethicist) to address similar cases earlier in the ICU stay. The GOC RRT is a unique concept, given that it supplements the normal ACP and goals of care conversations, developed on the basis of research by palliative care physicians $(7 ; 8)$. The aim is to bring different members of the team together early on in an ICU admission to facilitate a conversation with patients and their surrogate decision-makers on their goals during hospitalisation. These conversations have proven to be highly effective, and they have now become one of the strategic focus points of the institution where the ethicist is serving.

The benefit this approach has for patients is underwritten by the principle of solidarity. Solidarity refers to informing the obligations of role players with a focus on the common good of the group in their particular interactions. In this case, it was important for the healthcare team to realise that they had an obligation, as did Mr BM, to focus on the common good (minimising suffering) and to be cognisant of the unique context of the situation (COVID challenges and complicated grief). The different parties also had to realise that they were connected and interdependent and must collectively seek to achieve the value of justice and the common good. Focusing on our interdependencies benefits not only the parties to an ethical conflict, but also the cause of solidarity (9).

Competing interests and funding support: None declared. 


\section{References}

1. Zwakman M, Pollock K, Bulli F, Caswell G, erv B, van Delden JJM, et al. Trained facilitators' experiences with structured advance care planning conversations in oncology: an international focus group study within the ACTION trial. BMC Cancer. 2019;19(1):1026.

2. Wichmann $A B$, van Dam $H$, Thoonsen $B$, Boer TA, Engels $Y$, Groenewoud AS. Advance care planning conversations with palliative patients: looking through the GP's eyes. BMC Fam Pract. 2018;19(1):184.

3. Saeid Y, Salaree MM, Ebadi A, Moradian ST. Family intensive care unit syndrome: an integrative review. Iran J Nurs Midwifery Res. 2020;25(5), 361-368. DOI: 10.4103/ijnmr.IJNMR_243_19

4. Hardy NK, Nortjé N. Facilitated conversation: a useful tool in an ethicist's toolbox. Can J Bioeth. 2020;3:175-7. DOI: 10.7202/1070291ar

5. McAndrew NS, Leske J, Schroeter K. Moral distress in critical care nursing: the state of the science. Nurs Eth. 2018;25(5):552-570.
6. An Act Relating to General Procedures and Requirements for Certain Do-Not-Resuscitate Orders; Creating a Criminal Offense. Texas Senate Bill 11 (2017). [cited 2021 April 19]. Available from: https:// capitol.texas.gov/tlodocs/851/billtext/pdf/ SB00011F.pdf\#navpanes $=0$

7. Khandelwal N, Curtis JR, Freedman VA, Kasper JD, Gozalo P, Engelberg RA, Teno JM. How often is end-of-life care in the United States inconsistent with patients' goals of care? J Palliat Med. 2017;20(12): 1400-1404.

8. Epstein RM, Duberstein PR, Fenton JJ, Fiscella K, Hoerger M, Tancredi DJ, et al. Effect of a patient-centered communication intervention on oncologist-patient communication, quality of life, and health care utilization in advanced cancer. JAMA Oncol. 2017;3(1):92-100.

9. Scholz S. Solidarity. In: Ten Have H, editor. Encyclopedia of Global Bioethics. Dordrecht: Springer International; 2016. p. 2652-2658. 